\title{
QUALIDADE DE VIDA DE IDOSOS SEGUNDO A PRÁTICA REGULAR DE EXERCÍCIO FÍSICO
}

\section{QUALITY OF LIFE OF ELDERLY PEOPLE ACCORDING TO REGULAR PHYSICAL EXERCISE PRACTICE}

\section{CALIDAD DE VIDA DE LOS ADULTOS MAYORES SEGÚN LA PRÁCTICA REGULAR DE EJERCICIO FÍSICO}

\author{
Bruna Ferreira Silva ${ }^{1}$, Mariana Rosa Ribeiro Cardoso ${ }^{2}$, Grace de Sousa Lopes ${ }^{3}$, Joyce Mara \\ Gabriel Duarte ${ }^{4}$, Adriana Cristina Nicolussi ${ }^{5}$
}

Como citar esse artigo: Silva BF, Cardoso MRR, Lopes GS, Duarte JMG, Nicolussi AC. Qualidade de vida de idosos segundo a prática regular de exercício físico. Rev Enferm Atenção Saúde [Internet]. 2021 [acesso em:__]; 10(3):e202132. doi: https://doi.org/ 10.18554/reas.v10i3.4566

\section{RESUMO}

Objetivo: verificar a qualidade de vida de idosos segundo a prática regular de exercício físico. Método: estudo transversal realizado com 63 idosos cadastrados em uma Unidade Básica de Saúde de um município no interior de Minas Gerais. Os dados foram coletados de maio a setembro de 2019 com instrumentos validados e realizadas as análises: descritiva e bivariada (Teste t de Student) $(\mathrm{p}<0,05)$. Resultados: Os idosos que praticavam exercício físico regular apresentaram maior média nos domínios: físico $(p<0,001)$, relações sociais $(p=0,002)$ e meio ambiente $(\mathrm{p}<0,001)$; tal como na autoavaliação da qualidade de vida $(\mathrm{p}=0,001)$; na satisfação com a própria saúde $(\mathrm{p}=0,003)$ e no escore total de qualidade de vida $(\mathrm{p}<0,001)$ quando comparados àqueles que não tinham esse hábito de vida. Conclusão: a prática de exercícios físicos regulares pode contribuir para uma melhor qualidade de vida da população idosa.

Descritores: Qualidade de Vida; Saúde do Idoso; Exercício Físico.

\footnotetext{
${ }^{1}$ Uberlândia Medical Center - Unidade hospitalar. Enfermeira. Residente de Enfermagem em Saúde do Idoso, Universidade Federal do Triângulo Mineiro (UFTM). Uberaba, MG, Brasil. http://orcid.org/0000-0001-8443-2481 ${ }^{2}$ Enfermeira. Residente de Enfermagem em Saúde do Idoso, Mestranda do Programa de Pós-graduação em Atenção à Saúde da Universidade Federal do Triângulo Mineiro (UFTM). Uberaba, MG, Brasil. http://orcid.org/0000-0002-8358-0818

${ }^{3}$ Universidade Federal do Triângulo Mineiro (UFTM). Enfermeira. Residente de Enfermagem em Saúde do Idoso, Universidade Federal do Triângulo Mineiro (UFTM). Uberaba, MG, Brasil. http://orcid.org/0000-00021549-1135

${ }^{4}$ Universidade Federal do Triangulo Mineiro (UFTM). Enfermeira. Doutora em Atenção à Saúde. Enfermeira no Centro de Educação Profissional (CEFORES) da Universidade Federal do Triangulo Mineiro (UFTM). Uberaba, MG, Brasil. http://orcid.org/0000-0002-4501-9712

${ }^{5}$ Universidade Federal do Triângulo Mineiro (UFTM). Enfermeira, Doutora em Ciências. Professor Adjunto do Departamento de Enfermagem na Assistência Hospitalar do Curso de Graduação em Enfermagem da UFTM. http://orcid.org/0000-0001-5600-7533
} 


\begin{abstract}
Objective: to verify the quality of life of the elderly people according to regular physical exercise practice. Method: cross-sectional study carried out with 63 elderly people registered in a Basic Health Unit in a city in the interior of Minas Gerais. Data were collected from May to September 2019 with validated instruments and analyzes were performed: descriptive and bivariate (Student's t test) $(\mathrm{p}<0.05)$. Results: the elderly who practiced regular physical exercise had higher averages in the physical $(p<0.001)$, social relationships $(p=0.002)$, environment $(\mathrm{p}<0.001)$ domains; as in the self-assessment of quality of life $(\mathrm{p}=0.001)$; satisfaction with their own health $(\mathrm{p}=0.003)$, and quality of life total score $(\mathrm{p}<0.001)$ when compared to those who did not have this habit of life. Conclusion: the practice of regular physical exercises can contribute to a better quality of life for the elderly population.
\end{abstract}

Descriptors: Quality of Life; Health of the Elderly; Exercise.

\title{
RESUMEN
}

Objetivo: analizar la calidad de vida de los adultos mayores según la práctica regular de ejercicio físico. Método: estudio transversal realizado con 63 adultos mayores inscritos en una Unidad Básica de Salud de una ciudad del interior de Minas Gerais. Los datos se recolectaron de mayo a septiembre de 2019 con instrumentos validados y se realizaron los análisis: descriptivos y bivariados (prueba t de Student) ( $\mathrm{p}<0,05)$. Resultados: los adultos mayores que practicaban ejercicio físico regularmente presentaron promedios más altos en los dominios: físico $(\mathrm{p}<0,001)$, relaciones sociales $(\mathrm{p}=0,002)$ y medio ambiente $(\mathrm{p}<0,001)$; y en la autoevaluación de la calidad de vida $(\mathrm{p}=0,001)$; satisfacción con su salud $(\mathrm{p}=0,003)$ y puntaje total de calidad de vida ( $<<0,001$ ); en comparación con los que no tenían ese hábito de vida. Conclusión: la práctica de ejercicio físico regular puede contribuir a que la población de adultos mayores tenga una mejor calidad de vida.

Descriptores: Calidad de Vida; Salud del Adulto Mayor; Ejercicio Físico.

\section{INTRODUÇÃO}

Dentre diversos grupos existentes no meio social, a população idosa vem crescendo exponencialmente no Brasil e no mundo. Atualmente a expectativa de vida do brasileiro é de 75,8 anos com variações entre os sexos. Essa transição demográfica com queda da mortalidade deve-se à incorporação de avanços da medicina e às políticas de saúde pública. ${ }^{1}$

O envelhecimento acarreta mudanças e influencia na qualidade de vida (QV) do idoso. O exercício físico pode ser utilizado como método para melhorar e contribuir nesse processo e trazer inúmeros benefícios, ajudando na cognição, função respiratória e cardíaca, força muscular, além de prevenir o surgimento de doenças, colaborando assim, para uma melhor QV. ${ }^{2}$

A QV dos idosos pode variar conforme a região e o contexto cultural em que se encontram. Em um estudo, verificou-se que a faixa etária com melhor QV em geral é de 60 a 69 anos, reduzindo a cada década; consequentemente, menos da metade dos idosos com 80 anos ou mais apresentaram nível adequado de QV. ${ }^{3}$ Com o envelhecimento da população, o Estado deve estar preparado para garantir uma atenção integral, reconhecendo as 
características e especificidades dos idosos, consagrando sua QV. ${ }^{4}$

A avaliação da QV é uma forma de considerar os diversos aspectos e circunstâncias do contexto que o indivíduo está inserido. Ela analisa o ser na sua integralidade como as condições de saúde física, psicológica, independência em atividades cotidianas, relações com a sociedade, meio ambiente e espiritualidade. $^{5}$ Este termo é frequentemente estudado, sendo compreendido como uma condição eminente da humanidade, onde aborda também aspectos como vida familiar, amorosa, social e ambiental, sendo um constructo amplo com múltiplos significados. $^{6}$

A QV pode ser definida como a "percepção do indivíduo de sua posição na vida no contexto da cultura e sistema de valores nos quais ele vive e em relação aos seus objetivos, expectativas, padrões e preocupações", considerando a natureza do ser que está inserido em um cenário cultural e social, levando em conta outros fatores como experiências já vividas, condições de saúde e meio ambiente. ${ }^{7}$

Com o intuito de mensurar a QV dos indivíduos, existem vários questionários validados, dentre eles, o World Health Organization Quality of Live-bref (WHOQOL-BREF), que foi desenvolvido pela $\mathrm{OMS}^{7}$ e validado no Brasil. ${ }^{8}$
Um estudo apresentou os benefícios adquiridos com a prática regular de atividade física nos domínios físico, mental e social de idosas, e esta prática foi condicionante para um envelhecimento saudável e melhor QV. ${ }^{9}$ Assim sendo, o uso de um instrumento validado irá amparar os resultados a serem investigados.

Com a hipótese de que a prática regular do exercício físico influencia positivamente a QV dos idosos, considerase importante realizar pesquisas que visem confrontar resultados entre idosos que realizam com os que não realizam exercícios físicos. Conhecer tais informações é importante para auxiliar a equipe de saúde no incentivo à prática de exercícios físicos aos idosos durante a assistência na atenção primária. Diante disto, o presente estudo tem como objetivo verificar a qualidade de vida de idosos segundo a prática regular de exercício físico.

\section{MÉTODO}

Trata-se de um estudo quantitativo, de delineamento descritivo, exploratório e transversal, realizado em uma Unidade Básica de Saúde (UBS) de uma cidade do interior de Minas Gerais.

Foi realizado amostragem nãoprobabilística de conveniência, ou seja, foram convidados a participar do estudo 
idosos que compareceram na referida UBS. Os critérios de inclusão foram: ter idade maior ou igual a 60 anos e residir na área de abrangência da referida UBS. Foram excluídos os idosos com doenças neurológicas e condições físicas com quadro de dependência funcional que pudesse dificultar a participação na pesquisa.

A amostra final foi composta por 63 idosos, dos quais 23 compuseram o grupo que praticava exercício físico (como por exemplo: ginástica e atividades de circuito) com regularidade (ao menos duas vezes por semana, há três meses); e 40 idosos foram alocados para o grupo que não praticava exercício físico. Ressaltando que exercícios físicos são práticas regulares, o que diferencia de atividade física que é aquela que não requer regularidade $\mathrm{e}$ monitorização por profissional, como atividades cotidianas, por exemplo. ${ }^{2}$

A coleta de dados ocorreu de forma individual, nos dias em que os idosos compareciam a UBS para acompanhamento médico, no período de maio a setembro de 2019. Foi aplicado um questionário para coleta de dados sociodemográficos, como idade, sexo, cor/raça, nível de escolaridade e religião.

Em seguida, utilizou-se o instrumento WHOQOL-BREF, que é uma versão abreviada do WHOQOL-100, cujas respostas devem ser embasadas nas últimas duas semanas antes do ato da entrevista. $\mathrm{O}$ instrumento é constituído por 26 questões, sendo duas perguntas gerais e as demais compõem quatro domínios: físico, psicológico, relações sociais e meio ambiente. $^{8}$

Com relação às questões gerais, a primeira refere-se à autoavaliação de QV e tem como opções de resposta: "muito ruim", "ruim", "nem ruim nem boa", "boa" e "muito boa". A segunda avalia a satisfação em relação à própria saúde, cujas opções são: "muito insatisfeito", "insatisfeito", "nem satisfeito nem insatisfeito", "satisfeito" e "muito satisfeito". 8

Para a análise dos dados, foi utilizado o PSPP Statistical Analysis Software versão 1.2.0 - 2018. Foi utilizado o teste de Kolmogorov-Smirnov para testar a normalidade, realizado cálculo das frequências absoluta e relativa das variáveis sociodemográficas e cálculo de média e desvio padrão dos domínios do WHOQOL-BREF após a sintaxe do mesmo. Foi realizado o Teste de Levene para Igualdade de Varianças e a análise estatística inferencial pelo Teste $\mathrm{t}$ de Student, a fim de certificar a existência de diferenças significativas entre os grupos pesquisados, sendo o intervalo de confiança adotado de $5 \%$, sendo $\mathrm{p}<0,05$.

O estudo foi aprovado pelo Comitê de Ética em Pesquisa da Universidade 
Federal do Triângulo Mineiro, número do Parecer 3.290.669 de 29/04/2019, em acordo à Resolução 466/2012 do Conselho Nacional de Saúde. ${ }^{10}$ Os idosos foram abordados pelas pesquisadoras assistentes, que explicaram a finalidade da pesquisa e que a participação seria totalmente voluntária e anônima; após o aceite, eles assinavam o Termo de Consentimento Livre e Esclarecido. Manteve-se o sigilo das informações, pois a identificação dos sujeitos foi realizada através de códigos.

\section{RESULTADOS}

Observa-se na tabela 1 que em ambos os grupos predominaram o sexo feminino, na faixa etária de 60 a 69 anos, cor/raça branca, religião católica e ensino fundamental incompleto.

Tabela 1. Características sociodemográficas dos idosos segundo a prática regular de exercício físico. Minas Gerais, Brasil, 2019.

\begin{tabular}{|c|c|c|c|}
\hline & Variáveis & $\begin{array}{c}\text { Idosos que praticavam } \\
\text { exercício físico } \\
\mathrm{n}(\%)\end{array}$ & $\begin{array}{c}\text { Idosos que não } \\
\text { praticavam } \\
\text { exercício físico } \\
\mathrm{n}(\%)\end{array}$ \\
\hline \multirow{2}{*}{ Sexo } & Feminino & $22(95,7)$ & $25(62,5)$ \\
\hline & Masculino & $1(4,3)$ & $15(37,5)$ \\
\hline \multirow{3}{*}{ Faixa Etária } & 60 a 69 & $13(56,5)$ & $24(60,0)$ \\
\hline & 70 a 79 & $9(39,2)$ & $14(35,0)$ \\
\hline & 80 ou acima & $1(4,3)$ & $2(5,0)$ \\
\hline \multirow{3}{*}{ Cor/Raça } & Branca & $10(43,4)$ & $24(60,0)$ \\
\hline & Mulata/ Parda & $8(34,8)$ & $14(35,0)$ \\
\hline & Negra & $5(21,8)$ & $2(5,0)$ \\
\hline \multirow{4}{*}{ Religião } & Católico & $9(39,1)$ & $23(57,5)$ \\
\hline & Espirita & $8(34,8)$ & $7(17,5)$ \\
\hline & Evangélico & $2(8,8)$ & $2(5,0)$ \\
\hline & Outras & $4(17,3)$ & $8(20,0)$ \\
\hline \multirow{5}{*}{$\begin{array}{l}\text { Nível de } \\
\text { escolaridade }\end{array}$} & Ensino fundamental incompleto & $11(47,9)$ & $28(70,0)$ \\
\hline & Ensino fundamental completo & $7(30,4)$ & $5(12,5)$ \\
\hline & Ensino médio incompleto & $1(4,3)$ & $3(7,5)$ \\
\hline & Ensino médio completo & $2(8,7)$ & $2(5,0)$ \\
\hline & Ensino superior completo & $2(8,7)$ & $2(5,0)$ \\
\hline
\end{tabular}

Fonte: Próprios Autores

A tabela 2 apresenta as médias e desvio padrão do questionário WHOQOLBREF para os dois grupos de idosos. É possível observar que os idosos que praticavam exercício físico regular apresentaram maior média nos domínios Rev Enferm Atenção Saúde [Online]. Out/Dez 2021; 10(3):e202132 físico $\quad(p<0,001), \quad$ relações sociais $(\mathrm{p}=0,002)$ e meio ambiente $(\mathrm{p}<0,001)$; na autoavaliação da qualidade de vida ( $\mathrm{p}=0,001)$; na satisfação com a própria saúde $(\mathrm{p}=0,003)$ e no escore total de qualidade de vida $(\mathrm{p}<0,001)$ quando 
comparados àqueles que não tinham esse

hábito de vida.

Tabela 2. Qualidade de vida de idosos segundo a prática regular de exercício físico. Minas Gerais, Brasil, 2019.

\begin{tabular}{|c|c|c|c|c|c|c|}
\hline \multirow[b]{2}{*}{ Variáveis } & \multicolumn{2}{|c|}{$\begin{array}{c}\text { Idosos que } \\
\text { praticavam exercício } \\
\text { físico }\end{array}$} & \multicolumn{2}{|c|}{$\begin{array}{c}\text { Idosos que não } \\
\text { praticavam exercício } \\
\text { físico }\end{array}$} & \multirow[b]{2}{*}{$\mathbf{t}^{*}$} & \multirow[b]{2}{*}{$\mathbf{p}^{* *}$} \\
\hline & Média & $\begin{array}{l}\text { Desvio } \\
\text { Padrão }\end{array}$ & Média & $\begin{array}{l}\text { Desvio } \\
\text { Padrão }\end{array}$ & & \\
\hline Autoavaliação da QV & 4,26 & 0,54 & 3,63 & 0,93 & 3,44 & 0,001 \\
\hline Satisfação com própria saúde & 3,91 & 0,73 & 3,15 & 1,05 & 3,07 & 0,003 \\
\hline Domínio Físico & 71,43 & 14,00 & 55,27 & 17,77 & 3.74 & 0,000 \\
\hline Domínio Psicológico & 73,01 & 11,84 & 67,19 & 16,69 & 1.61 & 0,113 \\
\hline Domínio Relações sociais & 75,36 & 13,64 & 60,83 & 19,17 & 3,19 & 0,002 \\
\hline Domínio Meio ambiente & 67,80 & 11,01 & 56,46 & 11,34 & 3,86 & 0,000 \\
\hline Escore total & 77,17 & 13,41 & 59,69 & 22,38 & 3,88 & 0,000 \\
\hline
\end{tabular}

*Teste t de Student. **p<0,05.

Fonte: Próprios Autores

A tabela 3 indica como os idosos autoavaliaram sua QV. A opção com mais alto percentual de resposta foi "boa" para ambos os grupos, contudo nota-se que no grupo de idosos que praticavam exercício físico, nenhum selecionou as opções "muito ruim" e "ruim".

Tabela 3. Autoavaliação da Qualidade de Vida dos idosos segundo a prática regular de exercício físico. Minas Gerais, Brasil, 2019.

\begin{tabular}{lcc}
\hline & $\begin{array}{c}\text { Idosos que praticavam exercício } \\
\text { físico } \\
\text { Categoria de respostas }\end{array}$ & $\begin{array}{c}\text { Idosos que não } \\
\text { praticavam exercício } \\
\text { físico } \\
\mathrm{n}(\%)\end{array}$ \\
\hline Muito ruim & $0(0,0)$ & $1(2,5)$ \\
Ruim & $0(0,0)$ & $3(7,5)$ \\
Nem ruim nem boa & $1(4,4)$ & $12(30,0)$ \\
Boa & $15(65,2)$ & $18(45,0)$ \\
Muito boa & $7(30,4)$ & $6(15,0)$ \\
\hline
\end{tabular}

Fonte: Próprios Autores

A tabela 4 demonstra como os idosos avaliaram a satisfação em relação à própria saúde. As opções "muito insatisfeito" e "insatisfeito" não foram respondidas por nenhum idoso do grupo que praticava exercício físico, cujo maior percentual de resposta foi para "satisfeito". Enquanto que para o grupo de idosos que não praticava exercícios, o maior percentual foi para a resposta "nem satisfeito nem insatisfeito". 
Tabela 4. Satisfação dos idosos em relação a sua própria saúde. Minas Gerais, Brasil, 2019.

\begin{tabular}{lcc}
\hline \multicolumn{1}{c}{ Categoria de respostas } & $\begin{array}{c}\text { Idosos que praticavam } \\
\text { exercício físico } \\
\mathrm{n}(\%)\end{array}$ & $\begin{array}{c}\text { Idosos que não praticavam } \\
\text { exercício físico } \\
\mathrm{n}(\%)\end{array}$ \\
\hline Muito insatisfeito & $0(0,0)$ & $3(7,5)$ \\
Insatisfeito & $0(0,0)$ & $7(17,5)$ \\
Nem satisfeito nem insatisfeito & $7(30,4)$ & $14(35,0)$ \\
Satisfeito & $11(47,8)$ & $13(32,5)$ \\
Muito satisfeito & $5(21,8)$ & $3(7,5)$ \\
\hline
\end{tabular}

Fonte: Próprios Autores

\section{DISCUSSÃO}

O maior percentual de idosos do sexo feminino condiz com outras pesquisas que avaliaram a QV de idosos segundo a prática de exercício físico ${ }^{11-12}$ e na faixa etária de 60 a 69 anos. $^{11}$

Com relação ao nível de escolaridade, esta pesquisa encontrou a maioria dos idosos com ensino fundamental incompleto. Já em estudo realizado em Goiás ${ }^{11}$, os idosos do grupo de exercícios foram semelhantes nas categorias de ensino fundamental, médio e superior que variou entre 30 a $35 \%$ em cada, enquanto que no grupo que não realizou exercício prevaleceu analfabeto e o ensino fundamental.

A prática regular de exercício físico tem ação positiva para uma QV melhor, como foi evidenciado na presente pesquisa, corroborando o estudo $^{2}$ cujos idosos praticantes de exercícios físicos obtiveram médias maiores nos quatro domínios do WHOQOL-BREF e nas facetas: funcionamento dos sentidos, autonomia e participação social do instrumento
WHOQOL-OLD, também com diferenças significativas em relação aos que não praticavam.

O exercício físico proporciona um envelhecimento ativo aumentando a QV dos idosos prevenindo que doenças cardiovasculares e sistêmicas venham a surgir, prolongando o tempo de vida saudável destes. ${ }^{13}$

Outro fator positivo é que idosos que realizaram exercícios físicos relataram sentirem-se mais autoconfiantes e seguros, colaborando com a autonomia, melhora das funções motoras do corpo e melhor relação social evitando o isolamento e doenças depressivas nessa fase de vida, contribuindo positivamente na QV. ${ }^{9}$

A prática de exercícios físicos para os idosos que participam de programas comunitários apresenta indicadores significativamente melhores principalmente quando é realizado o monitoramento dos exercícios levando em conta a frequência, duração e o tipo de treino realizado. ${ }^{14}$ Contudo, a prática de atividade física que é realizada 
cotidianamente, onde não são regulares e não demandam de acompanhamento do profissional, também demonstram resultados positivos melhorando $\mathrm{o}$ equilíbrio funcional do idoso. ${ }^{15}$

Estudo pesquisou a compreensão de idosos sobre efeitos da prática de exercícios físicos e encontrou que um grupo de idosos que treinava regularmente mostrou-se motivado à realização, buscando os efeitos positivos sobre a saúde e a sociabilização e demonstraram compreender os benefícios que um programa de exercício físico proporciona sobre a saúde física e mental. Estes idosos manifestaram sentimentos de bem-estar ao realizarem os movimentos físicos, favorecendo a autonomia para a realização das atividades de vida diária e melhor interação social. ${ }^{16}$

O sedentarismo, em especial para o idoso, é um dos principais fatores de risco para o desenvolvimento de doenças crônicas, como as doenças cardiovasculares que são preponderantes na população idosa. Essas doenças influenciam na diminuição da QV uma vez que reduz a capacidade funcional do indivíduo.

Estudo $^{11}$ comparou o escore total e as questões de autoavaliação da QV e satisfação com a própria saúde do WHOQOL-BREF e encontrou que os idosos ativos apresentaram médias superiores do que os idosos do grupo controle, ou seja, que não praticavam exercício físico, corroborando os achados desta pesquisa.

Uma revisão sistemática analisou 12 artigos e concluíram que os exercícios físicos, com ênfase no treinamento multicomponente com regularidade de duas a três vezes por semana, causaram efeitos benéficos para os idosos fragilizados nos aspectos físicos, cognitivos e na QV. ${ }^{17}$

De modo geral, quando se compara a QV entre os idosos que praticam exercício físico de forma regular com idosos sedentários, os que realizam exercício físico demonstram uma melhor $\mathrm{QV} .{ }^{18}$ Estudo mostrou que a QV do idoso depende em grande parte, da forma como ele compreende sua vida pessoal na singularidade do sujeito através dos fatores e possíveis eventos passados que influenciam diretamente sobre sua percepção de QV. ${ }^{2}$

Estudo, realizado com 97 idosos que realizavam atividades vinculadas às Unidades de Saúde, de Atenção ao Idoso e de Universidade, encontrou que a QV dos idosos foi considerada boa e acreditam que tais atividades coletivas devem ser estimuladas pelas políticas públicas visando atender as necessidades dos idosos. ${ }^{19}$ 
Os resultados deste estudo demonstram que a QV dos idosos pode ser influenciada pela prática de exercício físico, constatando a importância de se propiciar melhorias das políticas públicas e ações de educação em saúde voltadas para a população idosa. Observa-se que atualmente existe uma quantidade maior de pesquisas relacionadas ao envelhecimento, portanto é de suma relevância que estudos mais sofisticados e bem definidos sejam realizados. ${ }^{20}$

A prática de exercícios físicos pode proporcionar aos idosos um processo de envelhecimento mais saudável com maior interação social, preservação da cognição e melhor capacidade para a realização de suas atividades de vida diária.

Sabe-se que a prática do exercício físico regular é fundamental para $\mathrm{QV}$ biopsicossocial dos idosos, sendo uma atividade de baixo custo e alto impacto que colabora para inserção do indivíduo na sociedade, promove autonomia, aumenta autoestima e reduz doenças e agravos. É pertinente que os profissionais da saúde reconheçam a magnitude de estimular a prática de exercício físico regular em idosos durante consultas e ações de promoção a saúde.

\section{CONCLUSÕES}

$\mathrm{Na}$ avaliação da QV através do instrumento WHOQOL-BREF, os idosos que praticavam exercício físico regularmente apresentaram maiores médias em todos os domínios avaliados, com diferenças estatisticamente significativas para os domínios: físico, relações sociais, meio ambiente, escore total, na autoavaliação da QV e satisfação com sua própria saúde do que os idosos que não praticavam.

Como limitação, destaca-se a amostra pequena e de conveniência, pertencente à abrangência de uma única UBS da cidade, não permitindo a generalização dos resultados encontrados.

Contudo é substancial que a prática de exercícios físicos para os idosos seja incentivada como uma ação necessária para a prevenção e promoção para a saúde destes, sendo indispensável que políticas públicas motivem e invistam na prática regular de treinamento físico para esse grupo populacional.

\section{REFERÊNCIAS}

1. Agência IBGE Notícias. Expectativa de vida do brasileiro sobe para 75,8 anos. Rio de Janeiro: Agência IBGE Notícias; 2019 [citado em 26 abr 2020]. (Estatísticas Sociais). Disponível em: https://agenciadenoticias.ibge.gov.br/agenc ia-noticias/2019-agencia-denoticias/noticias/18469-expectativa-devida-do-brasileiro-sobe-para-75-8-anos. 2. Ferretti F, Beskow GCT, Slaviero RC, Ribeiro CG. Análise da qualidade de vida em idosos praticantes e não praticantes de exercício físico regular. Estud Interdiscip Envelhec. [Internet]. 2015 [citado em 03 
ago 2020]; 20(3):729-43. Disponível em: https://seer.ufrgs.br/RevEnvelhecer/article/ view/41384

3. Paiva MHP, Pegorari MS, Nascimento JS, Santos AS. Fatores associados à qualidade de vida de idosos comunitários da macrorregião do Triângulo do Sul, Minas Gerais, Brasil. Ciênc Saúde Colet. [Internet]. nov 2016 [citado em 26 abr 2020]; 21(11):3347-56. Disponível em: https://www.scielo.br/j/csc/a/dh9cYb85hH LVXNF3BH7Yykq/?format=pdf\&lang $=p t$ 4. Miranda GMD; Mendes, ACG; SILVA, ALA. O envelhecimento populacional brasileiro: desafios e consequências sociais atuais e futuras. Rev Bras Geriatr Gerontol. [Internet]. 2016 [citado em 26 maio 2020]; 19(3):507-19. Disponível em: https://www.scielo.br/j/rbgg/a/MT7nmJPP Rt9W8vndq8dpzDP/?format $=p d f \& l a n g=p t$ 5. Almeida-Brasil CC, Silveira MR, Silva KR, Lima MG, Faria CDCM, Cardoso CL et al. Qualidade de vida e características associadas: aplicação do WHOQOL-BREF no contexto da atenção primária à saúde. Ciênc Saúde Colet. [Internet]. maio 2017 [citado em 26 abr 2020]; 22(5):170516. Disponível em: https://www.scielo.br/j/csc/a/dh9cYb85hH LVXNF3BH7Yykq/?format=pdf\&lang $=p t$ 6. Portugal FB, Campos MR, Gonçalves DA, Mari JJ, Fortes SLCL. Qualidade de vida em pacientes da atenção primária do Rio de Janeiro e São Paulo, Brasil: associações com eventos de vida produtores de estresse e saúde mental. Ciênc Saúde Colet. [Internet]. fev 2016 [citado em 26 abr 2020]; 21(2):497508. Disponível em: https://www.scielo.br/j/csc/a/JTHZJ4XsGc yHJzgDxdfjRqC/?format=pdf\&lang=pt 7. Kuyken W, Orley J, Sartorius N, Power M, Herrman H, Schofield H, et al. The World Health Organization quality of life assessment (WHOQOL): position paper from the World Health Organization. Soc Sci Med. [Internet]. nov 1995 [citado em 26 abr 2020]; 41(10):1403-9. doi: http://dx.doi.org/10.1016/02779536(95)00112-k
8. Fleck MPA, Louzada S, Xavier M, Chachamovich E, Vieira G, Santos L, et al. Aplicação da versão em português do instrumento abreviado de avaliação da qualidade de vida "WHOQOL-bref". Rev Saúde Pública [Internet]. 2000 [citado em 26 abr 2020]; 34(2):178-83. Disponível em: https://www.scielo.br/j/rsp/a/JVdm5QNjj4 $\mathrm{xHsRzMFbF} 7 \mathrm{trN} /$ ?format=pdf\&lang $=\mathrm{pt}$ 9. Camboim FEF, Nóbrega MO, Davim RMB, Camboim JCA, Nunes RMV, Oliveira SX. Benefícios da atividade física na terceira idade para a qualidade de vida. Rev Enferm UFPE on line [Internet]. 2017 [citado em 26 abr 2020]; 11(6):241522. Disponível em:

https://periodicos.ufpe.br/revistas/revistaen fermagem/article/view/23405/19070 10. Conselho Nacional de Saúde (Brasil). Resolução No 466, de 12 de dezembro de 2012. Aprova as diretrizes e normas regulamentadoras de pesquisas envolvendo seres humanos. Brasília, DF: CNS; 2012. Disponível em:

https://conselho.saude.gov.br/resolucoes/2 012/Reso466.pdf

11. Costa FR, Rodrigues FM, Prudente COM, Souza IF. Qualidade de vida de idosos participantes e não participantes de programas públicos de exercícios físicos. Rev Bras Geriatr Gerontol. [Internet]. jan/fev 2018 [citado em 05 ago 2020]; 21(1):24-34. Disponível em:

https://www.scielo.br/j/rbgg/a/4FCHcrCpC vvhYjKdJVK8bqv/?format=pdf\&lang=pt 12. Vilaça AF, Pedrosa BCS, Amaral TCN, Andrade MA, Castro CMMB, França EET. O efeito do treinamento muscular inspiratório em idosos sobre a qualidade de vida, resposta imune, força muscular inspiratória e de membros inferiores: um ensaio clínico randomizado. Rev Bras Geriatr Gerontol. [Internet]. 2019 [citado em 05 ago 2020]; 22(6):e190157. Disponível em: https://www.scielo.br/j/rbgg/a/jDPDVtdyP KffQXhjcGx3NsG/?format=pdf\&lang=pt 13. Balbé GP, Benedetti TRB, Meurer ST, Mazo GZ. Fatores associados à baixa 
qualidade de vida de idosas praticantes de exercícios físicos. Estud Interdiscip Envelhec. [Internet]. 2016 [citado em 26 abr 2020]; 21(2):89-104. Disponível em: https://seer.ufrgs.br/RevEnvelhecer/article/ view/42743/44540.

14. Camões M, Fernandes F, Silva B, Rodrigues T, Costa N, Bezerra P. Exercício físico e qualidade de vida em idosos: diferentes contextos sociocomportamentais. Motricidade [Internet]. 2016 [citado em 02 set 2020]; 12(1):96-105. Disponível em: https://revistas.rcaap.pt/motricidade/article/ view/6301/7395

15. Ferreira MER, Cardoso GMP, Lima GD, Oliveira ALN. Treinamento resistido na qualidade de vida de idosos. Rev Saúde Meio Ambiente [Internet]. jan/jul 2019 [citado em 02 set 2020]; 8(1):52-62. Disponível em: https://fan.com.br/site/wpcontent/uploads/2019/07/treinamentoresistido-na-qualidade-de-vida-de-idososferreira-et-al-2019.pdf 16. Gomes Júnior, VFF, Brandão AB, Almeida FJM, Oliveira JGD. Compreensão de idosos sobre os benefícios da atividade física. Rev Bras Ciênc Saúde [Internet]. 2015 [citado em 26 abr 2020]; 19(3):193-8. Disponível em:

https://periodicos.ufpb.br/ojs/index.php/rbcs/ar ticle/view/25540/15098

17. Pillatt AP, Nielsson J, Schneider RH. Efeitos do exercício físico em idosos fragilizados: uma revisão sistemática. Fisioter Pesqui. [Internet]. abr/jun 2019 [citado em 05 ago 2020]; 26(2):210-7. Disponível em:
https://www.scielo.br/j/fp/a/HxHRwfLJ9N ZmkkDymvGRL4G/?lang=pt

18. Klein SK, Fofonka A, Hirdes A, Jacob MHV. Qualidade de vida e níveis de atividade física de moradores de residências terapêuticas do sul do Brasil. Ciênc Saúde Colet. [Internet]. maio 2018 [citado em 26 abr 2020]; 23(5):152130. Disponível em: https://www.scielo.br/j/csc/a/6YmCcmh5f GpJSM8JGYVc9XH/?format=pdf\&lang=p $\mathrm{t}$

19. França DZ, Shimano SGN, Martins LJP, Marcacine PR, Lima JC, Walsh IAP. Qualidade de vida em idosos participantes em atividades coletivas. In: I Seminário Envelhecimento Ativo: entre o aprender e o senescer [Internet]. 26-28 nov, 2018. Uberaba, MG; 2018 [citado em 26 maio 2020]. p. 55. (Revista de Enfermagem e Atenção à Saúde; vol. 7; n. 4). Disponível em:

http://seer.uftm.edu.br/revistaeletronica/ind ex.php/enfer/issue/view/212

20. Dawalibi NW, Anacleto GMC, Witer

C, Goulart RMM, Aquino RC.

Envelhecimento e qualidade de vida: análise da produção científica da SciELO. Estud Psicol. (Campinas) [Internet]. set 2013 [citado em 26 abr 2020]; 30(3):393-403. Disponível em: https://www.scielo.br/j/estpsi/a/Ygw4N4D Vy5DMVgLhGHLxydp/?format=pdf\&lan $\mathrm{g}=\mathrm{pt}$

RECEBIDO: $27 / 05 / 2020$

APROVADO: 05/02/2021

PUBLICADO: dez/2021 